\title{
LETTER \\ Active Contour Model Based on Salient Boundary Point Image for Object Contour Detection in Natural Image
}

\author{
Yan LI ${ }^{\dagger a)}$, Student Member, Siwei LUO ${ }^{\dagger}$, Member, and Qi ZOU ${ }^{\dagger}$, Nonmember
}

\begin{abstract}
SUMMARY This paper combines the LBP operator and the active contour model. It introduces a salient gradient vector flow snake (SGVF snake), based on a novel edge map generated from the salient boundary point image (SBP image). The MDGVM criterion process helps to reduce feature detail and background noise as well as retaining the salient boundary points. The resultant SBP image as an edge map gives powerful support to the SGVF snake because of the inherent combination of the intensity, gradient and texture cues. Experiments prove that the MDGVM process has high efficiency in reducing outliers and the SGVF snake is a large improvement over the GVF snake for contour detection, especially in natural images with low contrast and small texture background.

key words: Local Binary Pattern, active contour model, image segmentation, contour detection
\end{abstract}

\section{Introduction}

Object contour detection has been a field of great interest in recent years. It is the precondition for high-level vision activities such as object recognition and tracking. However, it is still an obstacle in computer vision. One solution for contour detection is active contour models which are usually applied in medical field to detect the organ contour in MR image. The object in medical images has mostly homogenous intensity, so the active contour models can gain good performance. However, the objects in natural images tend to be composed of parts with different brightness, different color and different texture, which makes the boundary detection more difficult.

Aiming at this, we present a novel contour detection method based on two parts. The first part is a combination of LBP (Local Binary Pattern) and maximal difference of texture to get a clear salient-boundary-point image (SBP image) with as less noises as possible. In the second part, in order to gain a closed outer contour of the object, we used a continuous boundary detection model, the salient gradient vector flow snake (SGVF snake for short) with novel edge map generated from the SBP image, and rewrote the energy function. The new edge map considers not only the gray value and gradient but also the texture change in local region, which give the snake model stronger support.

The remainder of this paper is organized as follows. The related work is in Sect. 2. The novel edge map and the formulation of SGVF snake are described in Sect. 3. Sec-

Manuscript received January 29, 2010.

Manuscript revised June 4, 2010.

${ }^{\dagger}$ The authors are with the Department of Computer Science Beijing Jiao Tong University, Postcode 100044 Beijing China.

a) E-mail: yan531@263.net

DOI: $10.1587 /$ transinf.E93.D.3136 tion 4 contains the experiments and discussion. We conclude in Sect. 5 .

\section{Related Work}

The method in this paper is closely related to three areas of current work.

The first area concerns the texture cues in natural image. The reference [1], [2] introduced the local binary pattern to describe the image features. In this paper, we use basic LBP operator as our boundary point detector. The threshold is a local brightness contrast operator, and the neighborhood indicates a texture operator. The combination of the low-level and middle-level features makes the method robust to small texture change.

The second area concerns the active contour model. The traditional parametric snake was proposed in 1987 by Kass et al [3]. The problems of initialization and poor convergence to boundary concavities are two key difficulties with the active contour model. An external force for snakes, called gradient vector flow (GVF) [4] was introduced to overcome the two key difficulties of snakes. It has been widely used and improved. And $\mathrm{Li}$ et al [5] proposed the vector field convolution (VFC) external force, which has better performance than the GVF snake in crossing the weak boundary.

The third area concerns the graph-cut-based methods [6]. Interactive segmentation such as matting [7] and graph cut [8] has wonderful performance of extracting objects in recent years. In these methods, the 'trimap' $T$ and the user interaction combine the position, color and shape cues, which provide strong prior to the extracting process. Compared to these methods, snake-based methods have higher degree of automation without user-defined knowledge, and have good performance in gray images especially in medical images.

\section{Formulation of LBP-Guide Snake}

In active contour models, the role of external force is to attract the deformable contour to the features of interest (FOI) in the image. Both traditional snake and GVF snake defined external energy to be a function of $|\nabla I|$. In this formulation, only the low-level image features, the gray value and gradient of the image, are used. If middle-level features such as texture or region cues participat in the active contour framework, the performance of snake will be improved. 


\subsection{Maximum Difference of Gray Value}

LBP operator can be an edge point detector because it effectively combined the intensity and texture information of the image. The $L B P$ value $L B P_{(x, y)}$ in $3 \times 3$ region around point $p(x, y)$ can be formulated as follows:

$$
\begin{aligned}
& \operatorname{LBP}_{(x, y)}=\sum_{i=-1}^{1} \sum_{j=-1}^{1} I^{\prime}(x-i, y-j) W(i, j) \\
& I^{\prime}(x-i, y-j)= \begin{cases}0 & \text { if }|I(x, y)-I(x-i, y-j)| \leq T H \\
1 & \text { if }|I(x, y)-I(x-i, y-j)|>T H\end{cases} \\
& W=\left[\begin{array}{ccc}
2^{5} & 2^{6} & 2^{7} \\
2^{4} & 0 & 2^{0} \\
2^{3} & 2^{2} & 2^{1}
\end{array}\right]
\end{aligned}
$$

Where $I(x, y)$ is the original image, $I^{\prime}(x, y)$ donates the change of the intensity among the center point and its neighborhood, $W$ is a transformation matrix, $T H$ is the threshold.

The LBP operator can transform the image to a texton map; however, it cannot give us a perfect boundary image. It is obviously that the SBP image in Fig. 1 (c) is much clearer than the LBP one in Fig. 1 (b), has less small veins. For a better definition, we represent them in binary pattern, where all the nonzero points are white. As an edge map for snake, the SBP image should have as less detailed features and background noises as possible; at the same time, retain the boundary points of the object as sufficient as possible. To realize this purpose, we need to reduce the useless points which are not the boundary points.

The basic idea is that the large change of features along a structure indicates the presence of a boundary segment. At each point lying on boundaries in LBP image, different texture forms appear on two sides along the boundary. One side is sparse while the other side is abundant as showed in four rectangles in Fig. 1 (b). Our method computes contrast value of the density of the two side points as our salient value. The density can be indicated as average intensity.

We defined the contrast of $p(x, y)$ as $\operatorname{cont}_{(x, y)}$ in:

$$
\operatorname{cont}_{(x, y)}=\max \left\{\operatorname{cont}_{(x, y)}^{C_{1}}, \operatorname{cont}_{(x, y)}^{C_{2}}, \ldots, \operatorname{cont}_{(x, y)}^{C_{n}}\right\}
$$

Where $C_{1}, C_{2}, \ldots, C_{n}$ are the different scales of areas, $\operatorname{cont}_{(x, y)}^{C_{i}}$ is the contrast value of gray value in a certain area scale. Figure $1(\mathrm{~d})$ is the illustration. The square point is

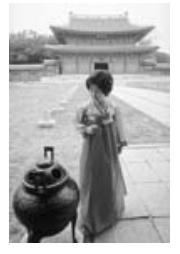

(a)OI

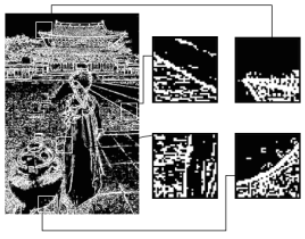

(b)LBP Image

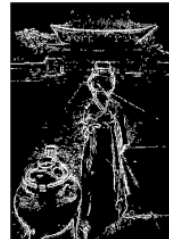

(c)SBP Image

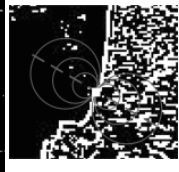

(d)MDGVM
Fig. 1 Different texture patterns beside of the boundary. a boundary point. The dashed line is the gradient direction, and the circles indicate the areas of different scales along the direction of the gradient. It is called MDGVM criterion (the maximum difference of gray value in multi-scale criterion): along the gradient direction of a point, in the same size area of the two sides, the maximum difference of gray value in unit area under different scales.

We can decide whether the point is a boundary point by a global constant threshold. If the constant is smaller, the SBP edge map is much clearer; in contrast the threshold is bigger, the SBP edge map is much blurrier. The SBP value of point $(x, y)$ can be defined as

$$
\operatorname{SBP}_{(x, y)}= \begin{cases}0 & \text { if } \operatorname{cont}_{(x, y)} \geq \operatorname{mean}(\operatorname{CONT}) \\ 1 & \text { if } \operatorname{cont}_{(x, y)}<\operatorname{mean}(\operatorname{CONT})\end{cases}
$$

Where mean is the average function, CONT is the contrast value matrix whose elements are $\operatorname{cont}_{(x, y)}$. Here, we choose mean $(C)$ as the constant threshold.

\subsection{Closed Contour Approximation}

The traditional calculation of the external force of the snake can be split to two steps: the formation of edge map form the image and the computation of external force from the edge map. The edge map $f(x, y)$ is generally a convolution of image gradient and a 2-D Gaussian functions as that given in (4)-(5), where $G_{\sigma}(x, y)$ is a 2-D Gaussian function with standard deviation $\sigma, *$ denotes linear convolution, and $\nabla$ denotes the gradient operator. The external force is typical an inverse of the edge map, so the quality of the edge map $f(x, y)$ is a critical factor in snake performance.

$$
\begin{aligned}
& f^{(1)}(x, y)=\left|\nabla\left[G_{\sigma}(x, y) * I(x, y)\right]\right|^{2} \\
& f^{(2)}(x, y)=G_{\sigma}(x, y) * I(x, y)
\end{aligned}
$$

The traditional edge map only takes low-level features such as luminance or gradient as consideration, which makes the edge map likely to be corrupt by the noise. And the region or configuration information among the image pixels is also lost. This information helps the boundary detection processes became more reliable and stable in higher level visual behaviors.

We propose a new static external force called salient gradient vector flow (SGVF) in this paper. The SGVF external force can combine the intensity, gradient and texture information, which will make the active contour model robust to small texture change in local region.

The SGVF external force is a SBP-based static force defined by the vector field $f_{S G V F}(x, y)=$ $\left[u_{S G V F}(x, y), v_{S G V F}(x, y)\right]$ that minimized the energy function:

$$
\begin{aligned}
E_{S G V F}= & \iint\left[\mu\left(\left|\nabla u_{S G V F}\right|^{2}+\left|\nabla v_{S G V F}\right|^{2}\right)\right. \\
& \left.+\nabla f_{S B P}{ }^{2}\left|f_{S G V F}-\nabla f_{S B P}\right|^{2}\right] d x d y
\end{aligned}
$$

Where $\mu$ is a parameter controlling the degree of smoothness of the GVF field, edge map $f_{S B P}(x, y)$ can be formulated as: 


$$
f_{S B P}(x, y)=G_{\sigma}(x, y) * S B P(x, y)
$$

where $G_{\sigma}(x, y)$ and $\sigma$ are the same as in (4)-(5), the definition of $\operatorname{SBP}(x, y)$ is in Eq. (3).

The novel edge map $f_{S B P}(x, y)$ generated from the SBP image contains much more information about the image than the traditional edge map. For contour detection in natural image, using a continuous boundary model SGVF snake, not a linear piecewise model [9], can leave out the contour grouping process and gain a simple closed object contour directly.

By replacing the standard external force by the SGVF field, and treating the snake $\boldsymbol{v}(s)$ as a function of time $\boldsymbol{v}(s, t)$, the iterative snake solution is Eq. (8). This equation can be solved numerically using identical finite difference approach of standard snakes.

$$
\frac{\partial \boldsymbol{v}(s, t)}{\partial t}=\alpha \boldsymbol{v}^{\prime \prime}(s, t)-\beta \boldsymbol{v}^{\prime \prime \prime \prime}(s, t)+\boldsymbol{f}_{S G V F}(\boldsymbol{v}(s, t))
$$

\section{Experiments}

The original images (OI for short) are random selected from the Berkeley segmentation dataset and benchmark. We discuss the appropriate $T H$ for the LBP progress and the validity of the MDGVM criterion. The Precision-Recall (P and $\mathrm{R})$ and F-value system in Eq. (9) is used to quantitate the performance of the SGVF snake. At the same time, to avoid the slight different of boundary location in different methods, we allow two-pixel difference beside the ground truth.

$$
F=P R / \alpha P+(1-\alpha) R, \alpha=0.5
$$

\subsection{Discussion of the LBP Threshold $T H$}

In the formation of SBP image, the threshold of LBP is the most important parameter. If the threshold is small, there will be lot of points beside the real boundary, which will stop the SGVF snake moving to the exact boundary location. And the edge map $f_{S B P}(x, y)$ may become more complicated. On the other hand, if the threshold is big, there may be some significant boundary points missing, this will make the snake deviate the real boundary.

The relation between the point-reduction and the threshold is a monotonic decreasing function as showed in Fig. 2. We need to find a corner point which has low computational complexity and at the same time retains the boundary points well. From the figure which shows data averaged from various natural images, the best threshold is 20 . In the following experiments, we use 20 as our LBP operator threshold.

\subsection{Validity of the MDGVM Criterion}

For gaining a clear object contour, we select the ground truth (GT for short) which has least and most significant boundaries. Figure 3 is the comparison of LBP and SBP image in

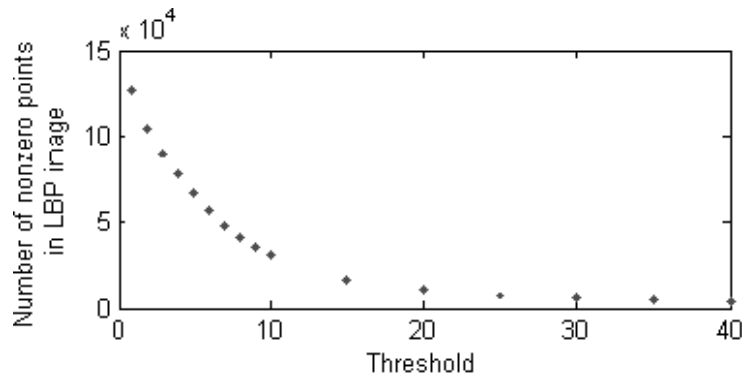

Fig. 2 Reduction of points along the threshold.

(a)
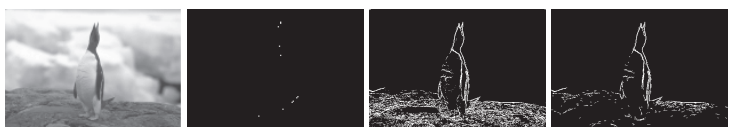

(b)

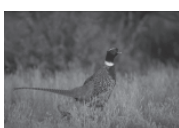

OI

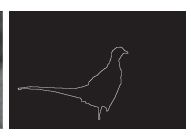

GT

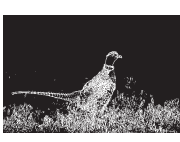

LBP Image

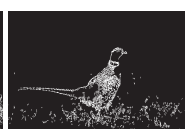

SBP Image

Fig. 3 The validity of the MDGVM criterion.

(a)
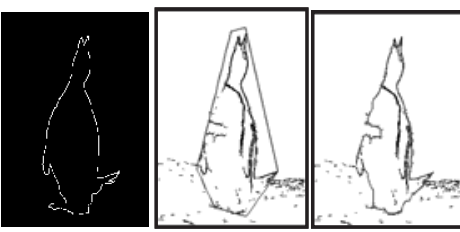

$F=0.65$

$\mathrm{P}=0.80, \mathrm{R}=0.55$

(b)
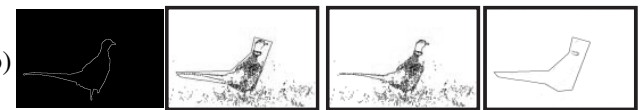

$$
F=0.53
$$$$
\mathrm{P}=0.65, \mathrm{R}=0.44
$$

SBP INI

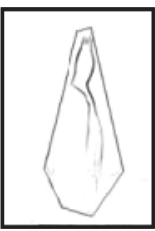

GT

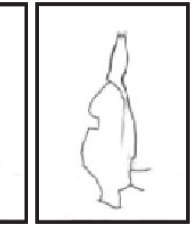

$F=0.56$

$\mathrm{P}=0.72, \mathrm{R}=0.46$

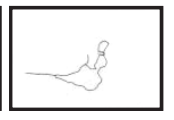

$\mathrm{F}=\mathbf{0 . 3 3}$

$\mathrm{P}=0.42, \mathrm{R}=0.27$

Fig. 4 Comparison between SGVF snake and GVF snake.

binary pattern. The average ratio of the quantity of points in LBP and SBP images is 1:0.296. More than 70\% points were reduced. And the average precision ratio in LBP image and SBP image is 1:2.173, increased nearly 1.2 times. This means that although the points get less, they belong to the real boundary get more. This makes the boundary more clear and integral.

\subsection{The Performance of SGVF Snake}

In Fig. 4, SBP INI and Gad INI are the same initialization on SBP-based edge map and gradient (Gad for short) edge map. The SGVF snake result with SBP INI and GVF result with Gad INI are labeled as SGVF and GVF respectively. The number of iteration in both snakes is 125 , and the parameter $\mu$ is 0.05 .

The Gad edge map is clear in high contrast region and 


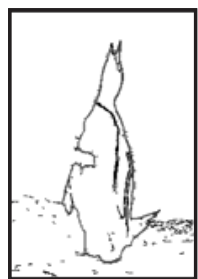

(a)SGVF

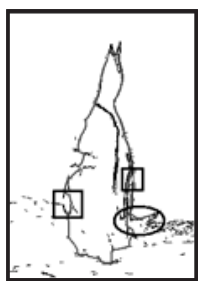

(b)SVFC

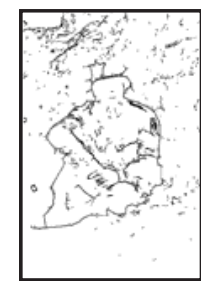

(c) SGVF

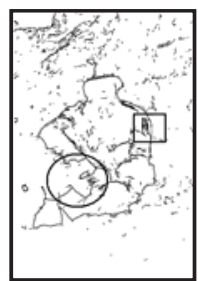

(d) SVFC
Fig. 5 Comparison of SVFC and SGVF snakes of cases in Fig. 4.

blurry in low contrast region because it only uses gradient as image cue. This results in that the GVF force is weak where the boundary is weak, so the tail and feet parts in case (a) shrinks in the GVF result, and the rostra part degenerates as a smooth curve. In case (b), there nearly has no strong edge, so the GVF performs badly. GVF model moves to the noise edges easily.

On the contrary, the SBP-based edge map is binary, so the SGVF force is strong enough to keep the corner well, such as the sharp on the rostra and tail parts in case (a) as well as the tail part in case (b). And edges have low contrast in Gad edge map may have high texture contrast in LBP image, which enhances the integrity of the object contour, and helps the model to avoid being distorted by noisy edges. So the contour gained by SGVF snake is more similar to the GT than that of the GVF snake, as the $F$ values show. This experiment proves that SGVF snake with SBP based edge map is very suitable for image with low contrast and small texture change.

\section{- Comparison between SGVF snake and SVFC snake}

We also test the performance of VFC snake with SBPbased edge map to prove that the GVF snake is more appropriate for combining with the SBP-based edge map. The VFC external force is an accumulation of GVF external force in local region essentially, which can improve the initialization sensitivity and noise obstruction to some extent. However, it always finds the strongest boundary in a local region, this makes it easily across the real boundary to the inner texture edges as the rectangles denote in Fig. 5. And at the boundary points missing part in SBP edge map, the VFC snake largely distorts due to its swift moving, which denoted by ellipses.

- Discussion about the SGVF snake and perceptual organization method

The method combining the edge detection approach and perceptual organization theory for gaining object boundary has precise biology and psychology theory basis, however, have insufficient performance. Firstly, the inputs of these methods are edges, and the grouping is a kind of clustering which only provide discrete boundary patches, not a single integrated closed contour. Secondly, in low gray contrast image, edge detection itself is a hard work, and the false of missing edges will be transferred and expanded in grouping stage.
On the contrary, the initial state of the SGVF snake is a closed integrated curve, which can improve the boundary detection performance in dark illumination and curtain situation, gain a refined closed contour. And in this paper, the initialization is roughly near the object contour which gives some prior, so the performance better than grouping methods is reasonable and predictable.

\section{Conclusion and Future Work}

This paper introduces a novel SGVF snake whose external force is generated from SBP-based edge map. This makes the snake powerful in synchronously processing the brightness and texture cues in natural image for contour detection. Experiments show the novel snake works well for contour detection especially in low contrast images.

However, there are also several problems unresolved. Firstly, missing of the boundary points will make the snake shrinks inside the object contour. Keeping high integrity of the real boundary points in SBP image is significant. Secondly, the linear texture form can not be well preserved in the MDGVM process. We need a method that can strengthen the salient value of linear texture form for better detection performance.

\section{Acknowledgments}

This work is supported by National Nature Science Foundation of China (60975078, 60902058, 60805041, 60872082, and 60773016), Beijing Natural Science Foundation (4092033) and Doctoral Foundations of Ministry of Education of China (200800041049).

\section{References}

[1] T. Ojala, M. Pietikäinen, and D. Harwood, "A comparative study of texture measures with classification based on feature distributions," Pattern Recognit., vol.29, pp.51-59, 1996.

[2] T. Ojala, M. Pietikäinen, and T.Mäenpää, "Multi-resolution gray scale and rotation invariant texture classification with local binary patterns," IEEE Trans. Pattern Anal. Mach. Intell., vol.24, no.7, pp.971-987, 2002.

[3] M. Kass, A. Witkin, and D. Terzopoulos, "Snakes: Active contour models," Int. J. Comput. Vis., vol.1, no.4, pp.321-331, 1987.

[4] C. Xu and J.L. Prince, "Snakes, shapes, and gradient vector flow," IEEE Trans. Image Process., vol.7, no.3, pp.359-369, 1998.

[5] B. Li and S.T. Acton, "Active contour external force using vector field convolution for image segmentation," IEEE Trans. Image Process., vol.16, pp.2096-2106, 2007.

[6] C. Rother, V. Kolmogorov, and A. Blake, "GrabCut-Interactive foreground extraction using iterated graph cut," ACM Trans. Graphics (SIGGRAPH'04), 2004.

[7] Y. Chuang, B. Curless, D. Salesin, and R. Szeliski, "A Bayesian approach to digital matting," Proc. IEEE Conf. Computer Vision and Pattern Recognition, 2001.

[8] V. Kwatra, A. Schold, I. Essa, G. Turk, and A. Bobick, "Graphcut textures: Image and video synthesis using graph cuts," Proc. ACM Siggraph, pp.277-286, 2003.

[9] Y. Li, S.W. Luo, and Q. Zou, "Learning to detect boundaries in natural image using texture cues and EM," Proc. Conf. Natural Computation, vol.4, pp.167-171, 2008. 